Revue d'histoire de l'enfance « irrégulière »

Le Temps de I'histoire

2| 1999

Cent ans de répressions des violences à enfants

Les perceptions des violences sexuelles commises sur enfants en Belgique (1830-1867) : construction juridique, pratique répressive et réactions sociales.

Geoffroy Le Clercq

2 OpenEdition

Journals

Édition électronique

URL : http://journals.openedition.org/rhei/33

DOI : $10.4000 /$ rhei.33

ISBN : 978-2-7535-1639-7

ISSN : $1777-540 \mathrm{X}$

Éditeur

Presses universitaires de Rennes

Édition imprimée

Date de publication : 15 novembre 1999

Pagination : 71-95

ISSN : 1287-2431

Référence électronique

Geoffroy Le Clercq, «Les perceptions des violences sexuelles commises sur enfants en Belgique

(1830-1867) : construction juridique, pratique répressive et réactions sociales. », Revue d'histoire de

l'enfance « irrégulière » [En ligne], 2 | 1999, mis en ligne le 30 juillet 2010, consulté le 04 décembre 2020.

URL : http://journals.openedition.org/rhei/33 ; DOI : https://doi.org/10.4000/rhei.33 


\section{Les perceptions des violences sexuelles commises sur enfants en Belgique (1830-1867) : construction juridique, pratique répressive et réactions sociales}

\section{Geoffroy Le Clercq ${ }^{(1)}$}

Les perceptions des violences sexuelles dans leurs multiples formes sont aussi variables que le sont les mentalités elles-mêmes. Des mentalités qui "n'existent jamais comme des structures planant au-dessus des réalités", mais qui " s'enracinent dans des temps, des lieux, des groupes et des êtres bumains». ${ }^{(2)}$ Qu'il s'agisse du regard du législateur, de ceux qui sont amenés à juger (magistrats, jurés, médecins) ou des familles des victimes, les sensibilités different et évoluent tout au long du XIXe siècle. Je vais tenter de les identifier, dans leur diversité.

Dans un premier temps, il convient d'examiner le "regard du droit" et du législateur. Durant la période étudiée, deux législations se succèdent en matière de violences sexuelles : le code pénal de 1810 et la loi du 15 juin 1846. Le processus qui a conduit au nécessaire élargissement des dispositions prévues dans le code de 1810 retiendra d'abord notre attention, laquelle se focalisera ensuite sur l'étude de la politique d'amendements menée par les parlementaires, s'attachant particulièrement à relever les conceptions particulières qui sous-tendent les modifications apportées au projet gouvernemental initial. Dans un second temps, je me livrerai à une analyse de la pratique répressive de la cour d'assises de Namur en matière de violences sexuelles pour la période 1830-1867. À nouveau, il sera question d'appréhender - à travers notamment la manière dont s'opère la qualification des faits - la façon dont les magistrats namurois perçoivent l'acte de violence sexuelle dans ses multiples formes, mais également l'auteur de l'acte et la victime. Les assises sont composées de deux corps importants : la cour proprement dite et le jury. Ce sont les jurés qui, dans les affaires jugées en assises, rendent le verdict, se prononçant sur la culpabilité ou l'innocence des prévenus. Cette coexistence, au sein d'une
(1) Assistant à

l'Université catholique de Louvain. Chercheur au Centre d'histoire du droit et de la justice.

(2) Mentalités. Histoire des cultures et des sociétés, Paris, 1989, (présentation de la revue par Robert Muchembled). 
(3) Ces procès d'assises sont conservés au palais de justice de Namur (PJN). Je précise que, sur les 39 affaires de violences sexuelles passées en jugement devant les assises namuroises de 1830 à 1867 (viols ou attentats à la pudeur), je n'ai pu en retrouver que 30 . même juridiction, de juges professionnels et de juges d'une session ne va pas sans causer certaines tensions. On remarque en effet d'indéniables différences de sensibilité entre les premiers et les seconds. Je m'attarderai dès lors également à mettre en exergue ces perceptions divergentes de la violence sexuelle. Enfin j'essaierai d'analyser des réactions sociales suscitées par la révélation de la violence sexuelle - avec, bien sûr, une insistance sur la spécificité de celles-ci lorsque la victime est encore un enfant - au sein de la sphère familiale et, plus globalement, dans les communautés villageoises du monde rural namurois. Pour ces deux dernières parties, une trentaine de procès de la cour d'assises namuroise (1830-1867) constituent la principale source. ${ }^{(3)}$ L'aire géographique couverte correspond au territoire que recouvre l'activité de cette juridiction, à savoir la province de Namur.

\section{1. - LE REGARD DE LA LOI ET DU LÉGISLATEUR : LA NORME EN MATIÈRE DE VIOLENCES SEXUELLES}

\section{a. - Le code pénal de 1810 et le malaise judiciaire}

Durant la période étudiée (1830-1867), deux législations en matière de violence sexuelle sont successivement en vigueur : il s'agit du très "utilitariste" code pénal de 1810 et de la loi du 15 juin 1846. Le code pénal présente de lourdes lacunes du point de vue de la protection légale apportée aux victimes d'abus sexuels. La plus grave déficience de la loi de 1810 trouve son expression dans la non incrimination de la "violence morale" : l'auteur d'un attentat à la pudeur commis sans violence matérielle sur un enfant n'est, jusqu'en 1846, passible d'aucune peine. La France comble cette lacune dès 1832. L'exemple français ne reste pas sans écho en Belgique. En 1834, deux projets de loi sont élaborés, mais rien de concret n'en ressort; ces projets restent lettre morte.

Ce n'est qu'en 1844 que le processus législatif est relancé. Il aura fallu pour cela l'épisode de la grave controverse suscitée par l'interprétation de l'article 334 du code pénal, épisode qui témoigne d'un profond malaise dans le monde judiciaire belge. Entre 1810 et 1842, certains 
magistrats accordent à l'article 334 du code pénal une portée qui dépasse les intentions primitives du législateur, afin que des actes jugés ignobles - des abus sexuels commis sans violence matérielle sur des enfants - puissent être réprimés. ${ }^{(4)}$ Cependant les trois cours d'appel du pays décident, dans le courant de 1842 et 1843, que l'article 334 vise le seul proxénète, adoptant ainsi une jurisprudence opposée à celle de la cour de cassation. L'idée défendue par les magistrats des cours d'appel est qu'il convient pour la magistrature d'éviter la confusion entre les rôles respectifs du "moraliste" et du "législateur". Au terme de la procédure du référé législatif, c'est la jurisprudence des cours d'appel que le parlement va consacrer par la loi du 31 mars 1844, préférant «le sens et la saine application des lois " à une interprétation moins restrictive de l'article 334.

\section{b. - Analyse "socio-législative" de la loi de 1846 : une "révolution" avortée}

Deux mois après la résolution de la controverse interprétative et dès lors que l'issue législative est devenue incontournable, le ministre de la justice d'Anethan ${ }^{(5)}$ dépose, devant la Chambre des représentants, un projet de loi destiné à remplacer les articles 331 à 335 du code pénal. Ce projet assez novateur suscite pas mal d'oppositions, comme en témoignent les rapports des commissions parlementaires. Ces débats permettent de déceler les préoccupations profondes d'hommes politiques de tendances différentes, dans leur complexité et leurs divergences, de même qu'un certain discours sur la sexualité et une conception particulière de l'ordre public.

Avant de passer à cette analyse, il me semble intéressant de réserver quelques lignes à une description du "climat" dans lequel vont évoluer les débats et le processus qui conduira à l'adoption définitive de la loi du 15 juin 1846. Chaque fois que le projet est à l'ordre du jour d'une séance, les tribunes sont évacuées et la Chambre se forme en comité secret. C'est encore à huis clos que se réunissent les commissions parlementaires qui débattent des articles du projet et pour lesquelles nous disposons de rapports dans les Annales parlementaires. Cet inhabituel secret est lié à la nature des sujets qui sont discutés : violence sexuelle,
(4) M. VINCINEAU,

La Débauche en Droit et le droit à la débauche, Bruxelles, 1985, p. 28. Article $334 \mathrm{du}$ code pénal : "Quiconque aura attenté aux mours, en excitant, favorisant, facilitant habituellement la débauche ou la corruption de la jeunesse de l'un ou de l'autre sexe au-dessous de l'âge de 21 ans, sera puni d'un emprisonnement de 6 mois à 2 ans et d'une amende de 50 francs à 500 francs ».

Contrairement à ce qui était initialement prévu, la portée de l'article n'était pas limitée au seul proxénétisme, mais étendue plus largement à la répression de la séduction des mineurs en vue de satisfaire son propre vice.

(5) Jules d'Anethan (1803-1888) est successivement avocat, juge d'instruction et avocat général près la cour 
d'appel de Bruxelles.

Parmi ses fonctions politiques, il est notamment ministre de la justice de 1843 à 1847.

(6) Ainsi, cette remarque du député

P. Devaux : « des projets de loi qui n'ont trait ni à un intérêt matériel, $[. .$.$] ni$ à un intérêt du fisc, ni à un intérêt politique, obtiennent difficilement leur tour de discussion. La loi dont nous nous occupons dans ce moment a déjà été plusieurs fois à l'ordre du jour sans pouvoir être discutée ». Annales parlementaires, Chambre des représentants, séance du 5 décembre 1845, p. 178.

(7) À part une modification minime, les deux premiers articles du projet de loi de d'Anethan reproduisent identiquement les articles 331 et 332 du code pénal de 1810.

(8) La Belgique judiciaire, (Gazette des tribunaux belges et étrangers), t. III, Bruxelles, 1845, p. 289. proxénétisme. La sexualité, une réalité dont on parle certes, mais à mimot et en prenant garde qu'elle ne soit entendue par n'importe qui. Dans ce siècle puritain, les "choses du sexe" sont honteuses. Il semble que le malaise se double en outre d'un certain désintérêt pour une matière que certains jugent futile. ${ }^{(6)}$ Ces observations permettent de mieux comprendre pourquoi, pendant près de 10 ans, suite aux projets de Haus et Lebeau, le processus législatif destiné à améliorer la législation en vigueur est resté en suspens.

L'essentiel du travail de sélection et des modifications apportées aux articles du projet de loi de d'Anethan a été réalisé par la commission de travail de la Chambre des représentants, celle du Sénat n'ayant été qu'une chambre d'entérinement. Cette commission est composée de six députés, catholiques et libéraux, mais il est difficile de déterminer dans quelle proportion. Il est également incertain, dans l'étude des argumentations, de rendre à chaque discours sa tendance politique, vu que la commission se réunit toujours en comité secret et que les rapports dont nous disposons respectent l'anonymat des députés qui s'y sont exprimés. Ceci rend malheureusement plus incertaine l'identification des positions spécifiquement liées à une tendance catholique ou libérale. Mon but n'est pas de me livrer ici à une analyse exhaustive - article par article des débats ayant émaillé la progressive élaboration de la nouvelle législation. Je me limiterai à l'étude des discussions suscitées par un seul des articles du projet de loi du ministre de la justice d'Anethan, l'article 3 :

"Si le fait repris aux deux articles précédents ${ }^{(7)}$ [il s'agit des viols, tentatives de viol et attentats à la pudeur] a été commis sans violence, l'auteur encourra néanmoins la même peine, s'il n'est parvenu à le commettre que par abus d'autorité ou de pouvoir, ou par l'emploi de moyens frauduleux, tels que la victime a été mise dans l'impossibilité soit de donner un consentement libre, soit d'opposer de la résistance ". ${ }^{(8)}$

Précisons deux choses : d'une part, que ces peines sont celles des travaux forcés dans le cas de victimes de moins de 15 ans et de la réclusion pour les victimes dites adultes (de 15 ans et plus); d'autre part, que les moyens frauduleux dont il est question peuvent par exemple consister en l'administration d'une drogue à la victime. 
L'article 3 est accepté tel quel par un seul des 6 députés. Deux autres parlementaires sont prêts à adopter l'article pour autant que soient retranchés les termes "par abus d'autorité et de pouvoir». La justification qu'ils donnent de ce rejet est fort savoureuse : prendre "l'abus d'autorité de pouvoir comme conditions essentielles du crime [...] exposerait la société à de grands dangers". L'on craint que "fréquemment, par des spéculations honteuses, des individus qui se trouvent dans la condition de domesticité ne consentissent à l'acte vénérien avec des enfants de famille, et ne vinssent ensuite impudemment déclarer, en produisant quelques témoins, que ce consentement n'a été arraché que par l'abus d'autorité dont on s'est rendu coupable à leur égard». ${ }^{\text {9) }}$

Ici, il est bien clair que les députés de la commission se rangent presque unanimement (ils sont en effet cinq sur six à rejeter ces termes) sous la bannière d'une conscience de classe aigüe, la leur, pour protéger ses intérêts. Admettre un tel article serait ouvrir la porte à des poursuites judicaires qui pourraient éclabousser de honte et de scandale ceux-là mêmes qui s'érigent en modèle (au moins apparent) de rigueur morale. Comme l'indiquent Aron et Kempf, la bourgeoisie confie à la morale le soin de "l'ennoblir" et "s'efforce uniformément à la respectabilité ». ${ }^{(10)}$ Cette préoccupation de défense "d'intérêts de classe" représente un guide puissant dans la politique d'amendements qui seront réalisés en commission.

La question de l'article 3 amputé des mots "par abus d'autorité et de pouvoir » divise la commission en deux camps : trois membres sont prêts à l'accepter avec cette suppression, trois autres s'y opposent toujours. Selon ces derniers, les mots «emploi de moyens frauduleux, tels que la victime de l'attentat a été mise dans l'impossibilité, soit de donner un consentement libre, soit d'opposer de la résistance, sont d'une élasticité telle que souvent les officiers du ministère public seraient exposés à franchir la limite qui sépare en cette matière l'acte immoral du fait punissable, en confondant avec les actes de violence et de corruption tous les actes de libertinage, toutes les immoralités [...] que la société et la personne qui en a été l'objet n'ont souvent pas intérêt à voir punir tant qu'ils ne dégénèrent pas en scandale public ». Ces membres de la commission ajoutent qu' «ils ne veulent pas non plus donner aux organes de la loi et de la société la mission de constater de pareils actes, parce que ce serait livrer la vie privée à une intolérable inquisition " et qu'" avec de pareilles dispositions
(9) La Belgique judiciaire, op. cit., p. 289.

(10) Jean-Paul ARON, Roger KEMPF, La Bourgeoisie, le sexe et l'honneur, Bruxelles, 1984, p. 17. 
(11) La Belgique judi-

ciaire, op. cit., p. 289.

(12) F. OST; M. Van de KERCHOVE, Bonnes mœurs, discours pénal et rationalité juridique. Essai d'analyse critique,

Publications des facultés universitaires SaintLouis, Bruxelles, 1981, p. 49 .

(13) Ibid.

(14) Le plus grand danger ne serait-il pas, au bout du compte, que la réputation d'un homme soit injustement bafouée, quand il n'aurait, à travers ses actes, que réalisé les secrets désirs d'une prétendue victime qui, après coup, prétexterait une soitdisante machination?
[...] le simple désordre deviendrait un délit et que la publicité des poursuites bouleverserait les familles sans guérir les maux $»{ }^{(11)}$

Premier élément fort intéressant à mettre en exergue parmi les propos de ces députés, la distinction opérée entre l'acte immoral et le fait punissable. Deux niveaux de lecture sont ici possibles. On peut d'abord relever que la séparation du droit d'avec la morale et la religion est un des traits caractéristiques des législations du XIXe siècle. ${ }^{(12)}$ Cette tendance, consacrée dans le code pénal de 1810, est directement issue de la Révolution française qui y voit une des principales garanties de la liberté d'opinion en matière morale et religieuse. ${ }^{(13)}$ On peut émettre l'hypothèse que cette argumentation émane plus particulièrement de parlementaires de tendance politique libérale (anticléricaux). Ce que redoutent ces commissaires n'est-il pas, en quelque sorte, que la magistrature n'adopte le visage d'un nouveau clergé, en poursuivant toutes les immoralités et les actes de libertinage, qui ne sont pas pour autant des faits punissables. L'anticléricalisme, s'il s'agit bien de cela, se manifesterait ici également dans le choix non innocent des mots : on parle bien d'“intolérable inquisition". D'autre part, peut-être cette résistance à ces dispositions, cette crainte de confondre le fait punissable, l'acte de violence, avec les actes de libertinage (moralement mais non légalement condamnables) trouve-t-elle un autre fondement qui n'apparait pas ici formulé explicitement. Le ministre de la justice avait également proposé d'assimiler les jouissances sexuelles procurées avec ou à l'aide d'une personne atteinte d'aliénation mentale à un attentat à la pudeur commis avec violence. Mais la commission décide que, d'une part, la violence ne peut-être présumée et que, d'autre part, le fait de profiter d'une aliénée est " sans doute un acte infâme ", mais que "rien n'établit que cette personne n'a pas eu la conscience de son fait » et "qu'elle n'y a pas donné un secret assentiment ". Le classique préjugé de la femme secrètement consentante ressort explicitement, exprimé sans doute ici de façon d'autant moins détournée que la catégorie de victimes visée est la plus « sujette à caution ». Il est probable que ce genre de conception a également dû inciter les députés à rejeter le projet du ministre concernant la présomption de violence en cas d'usage de moyens frauduleux. ${ }^{(14)}$ 
Outre le danger de confusion entre les actes de libertinage et les faits punissables, le deuxième élément inacceptable est, selon les trois députés "réfractaires ", la menace que font peser ces dispositions sur l'ordre des familles. Et tout cela pour un résultat de toute façon néfaste. Irait-on risquer de ruiner des familles par d'honteuses révélations, alors même que "ni la victime ni la société n'y trouveront leur intérêt ". Pourquoi irait-on traquer le scandale, alors que, selon les termes de deux criminalistes français de renom, les actes " accomplis dans le secret [...] ne troublent point la société qui les ignore ". ${ }^{(15)}$ Il ressort de ce discours que le fait ne devient punissable qu'à partir du moment où il a engendré le scandale. En fait, si la violence sexuelle est perçue en soi comme un acte immoral, c'est le scandale qu'elle suscite par sa révélation publique qui lui confere le statut de fait punissable. Ceci parce que, révélée publiquement, elle jette sur la victime et sa famille un profond déshonneur. C'est cette dimension-là qui est perçue comme la plus dommageable. La violence sexuelle reste avant tout un acte moralement infâmant, pour son auteur bien sûr, mais aussi pour la victime et, par contagion, la famille entière de celle-ci.

Il faut ajouter que l'avènement de la société bourgeoise semble s'être accompagné de la constitution d'un "domaine privé", distinct d'un "domaine public", dont le noyau est formé par la famille, sphère d'intimité assez repliée sur elle-même ${ }^{(16)}$ et où, selon J. Poumarède, l'autorité du père est proclamée avec force. ${ }^{(17)}$ Un pater familias tout puissant donc, qui, au sein de la sphère familiale, constitue déjà en quelque sorte son propre juge et avec lequel, pourrait-on dire, la justice ne doit pas entrer en concurrence. Les mots de Chauveau et Hélie s'insèrent parfaitement dans cet ordre d'idées : il est, selon eux, exclu de pouvoir accepter «l'inquisition du magistrat dans la vie privée des citoyens, soumettre à ces investigations leurs actions intimes, ouvrir en un mot le sanctuaire du foyer domestique ". ${ }^{(18)}$

De l'analyse de la politique d'amendements menée par ces députés, il ressort clairement que les intérêts des victimes de violence sexuelle ne sont pas au centre de leur priorités. Volonté quasi unanime de protéger leur classe sociale, volonté pour d'autres d'éviter l'intrusion dans le "sanctuaire sacré de la famille" de magistrats "inquisiteurs", risquant de jeter sur lui un scandale dommageable, surtout pour l'honneur des
(15) Adolphe CHAU-

VEAU, Faustin HELIE, Théorie du Code pénal, 2ème éd., t. IV, Paris, 1843, p. 213.

(16) F. OST ; M. Van de KERCHOVE, op. cit., p. 52 .

(17) Jacques POUMAREDE., " L'Inceste et le droit bourgeois ", in Jacques POUMAREDE et Jean-Pierre ROYER, Droit, histoire et sexualité, Lille-Toulouse, 1987, p. 225.

(18) Adolphe CHAUVEAU, Faustin HELIE, op. cit., p. 214. 
pères; crainte sans doute également d'ouvrir la porte aux allégations de "pseudo-victimes" en réalité secrètement consentantes. Autant de préoccupations qui orientent les choix et qui postposent des innovations légales en avance sur leur temps. Dans la loi du 15 juin 1846, c'est l'article 3 qui constitue finalement l'avancée majeure :

"Sera puni de la réclusion, quiconque se rendra coupable d'un attentat à la pudeur, commis sans violence sur la personne ou à l'aide de la personne d'un enfant de l'un ou de l'autre sexe, âgé de moins de quatorze ans".

L'adoption de cet article n'a, pour ainsi dire, pratiquement pas suscité d'opposition, étant donné que la pratique judiciaire avait démontré son absolue nécessité. Désormais, tout individu ayant abusé sexuellement d'un enfant de moins de 14 ans est passible d'une peine de réclusion, même si celui-ci n'a pas opposé de résistance physique à l'acte commis sur lui. Cette nouvelle disposition constitue un premier pas vers une plus grande protection de l'enfance. Sans doute témoigne-t-elle également de l'émergence d'une plus grande sensibilité à l'égard de "l'enfance en danger".

\section{2. - LE REGARD DE LA JUSTICE : ANALYSE DE LA PRATIQUE RÉPRESSIVE DE LA COUR D’ASSISES DE NAMUR DE 1830 À 1867}

\section{* La pratique des magistrats}

L'étude d'une trentaine de dossiers criminels fait apparaître que les magistrats namurois sont en avance sur leur temps, apparemment sensibilisés au sort des victimes brisées par les abus sexuels et peut-être plus particulièrement, à celui des enfants. À ce propos, il est intéressant de signaler que, de bonne heure, les magistrats namurois ont utilisé l'article 334 du code pénal de 1810 (en réalité destiné à la répression du proxénétisme) pour essayer de combler les manquements de cette législation. Ainsi, en 1834 notamment, Gauthier, un jeune anachorète d'origine française est-il accusé et condamné par contumace pour attentats à la pudeur commis avec violence sur 13 garçons de moins de 15 ans, et également en vertu de l'article 334, pour " avoir attenté aux mours " à 
l'égard de 18 garçons de moins de 21 ans. Il ne s'agit ici en rien de proxénétisme, mais de faits qui seront incriminés, à partir de la loi du 15 juin 1846, comme "attentat à la pudeur sans violence».

Au plan légal, il faut savoir que, selon l'article 79 du code d'instruction criminelle, les enfants de moins de 15 ans sont entendus sans prestation de serment, par simple "forme de déclaration ${ }^{(19)}$ Dans leur qualification des faits, ceci n'empêche nullement les procureurs d'y accorder semble-t-il un véritable crédit, même lorsque, comme dans de nombreuses affaires, il n'y a, parallèlement au seul témoignage des enfants, aucune autre preuve tangible que les faits qu'ils décrivent ont bien eu lieu, et que ces faits sont antérieurs de plusieurs années à la mise en examen du prévenu. Cependant, ce qui dans ce genre d'affaires renforce la conviction des magistrats, c'est la multiplication de ces témoignages isolés, qui acquièrent de par leur nombre, toute leur crédibilité. Si, dans de pareilles circonstances (éloignement dans le temps et témoignage isolé de l'enfant), la tendance est en général à la qualification des faits en "attentat à la pudeur commis sans violence ", la "prise de risque " est parfois plus grande, quitte, pour les procureurs, à s'écarter de la stricte doctrine juridique. Examinons l'affaire Remacle. ${ }^{(20)}$

Thérèse Jaumotte est âgée de 13 ans lorsqu'elle se confie au juge d'instruction de ce qui lui est arrivé à peu près trois ans auparavant. Ce jour là, après l'avoir attirée chez lui, Remacle la couche à terre, met ses "parties » à nu. Il introduit son membre "assez profondément» en elle. L'enfant sort de chez l'inculpé en pleurant, sans que personne l'ait aperçue. Sa mère à qui l'enfant se confie directement après le viol confirme cette déposition, mais selon cette femme, les faits se sont déroulés quatre ans avant la mise en examen de Remacle. Elle explique que sa fille était "honteuse ", aussi ne la "visite »-t-elle pas. Des suites du viol, dit-elle, sa fille est malade pendant quinze jours. Les présomptions laissant à penser qu'un viol a bien été commis sur Thérèse sont réelles. Cependant, les faits remontent à un passé assez lointain, la mère n'a pas procédé à un examen des organes génitaux de sa fille et rien n'indique que Thérèse ait opposé une résistance matérielle à son agresseur. Une telle réaction, certes tout fait compréhensible de la part d'une si jeune
(19) J.-A. ROGRON, Code d'instruction criminelle, expliqué par ses motifs, par des exemples, par la jurisprudence, Bruxelles, 1851, p. 28.

(20) Palais de justice de Namur (PJN), Cour d'assises de Namur (CAN), affaire Remacle, le 28 août 1856 . 
(21) Le deuxième élément constitutif du crime de viol est la pénétration (il s'agit nécessairement de celle d'un pénis dans un vagin, la sodomie ne peut-être considérée comme viol).

(22) Code pénal, art. 2828.

(23) Adolphe CHAUVEAU, Faustin HELIE, op. cit.

(24) Un enfant, mais encore, par exemple, une jeune fille abusée par son père, une aliénée mentale...

(25) Dans l'affaire Bissot, le terme est utilisé par l'avocat général de Liège dans son acte d'accusation (24 février 1849). Du curé qui a commis des attouchements sur trois garçonnets, le magistrat dit : « respecter l'innocence, respecter le temple même de la religion, ce ne furent bientôt plus pour lui des devoirs sacrés "; PJN, CAN, affaire enfant, ne cadre pas avec la définition légale du viol, du moins selon la loi du 15 juin 1846, qui suppose nécessairement la violence comme élément constitutif du crime. ${ }^{(21)}$ Or cette violence peut être présumée, mais seulement à partir du moment où certaines "conditions" semblent remplies : il faudrait en principe que la victime d'un viol ait opposé une résistance " constante et toujours égale, car il suffit que cette résistance ait fléchi quelques instants pour faire présumer le consentement » (!), qu'elle ait également poussé des cris et appelé au secours, qu'elle porte des traces corporelles gageant de la force brutale à laquelle elle avait dû céder. ${ }^{(22)}$ Les deux criminalistes de renom Chauveau et Hélie reprennent ces "règles pleines de sagesse » aux anciens jurisconsultes, règles qu'ils qualifient $\mathrm{d}$ ' "utiles précautions pour conduire à la découverte de la vérité ". ${ }^{(23)}$ Ces "sages préceptes" véhiculent l'image stéréotypée d'une victime secrètement consentante qui, après une résistance feinte, se laisserait aller au "délice” de l'acte. Ils nous renvoient en outre à une grave insuffisance de la législation de la loi du 15 juin 1846 : lorsqu'un viol (une pénétration) est commis sur un enfant ${ }^{(24)}$ qui ne remplit pas ces "conditions idéales", le crime ne peut être - en principe - incriminé qu'en tant qu'attentat à la pudeur commis sans violence (matérielle). Pourtant, le crime de «viol consommé ou tenté avec violence " sur Thérèse Jaumotte est bel et bien retenu comme chef d'accusation contre Remacle. La qualification de ce crime représente donc une entorse par rapport à la stricte rigueur de la doctrine juridique. Face à un acte qu'ils considèrent tout à fait révoltant et dont la présomption qu'il ait bien eu lieu est grande, sans doute les magistrats jugent-ils en conscience ne pouvoir s'en tenir à la lettre de la loi. Cet exemple, que beaucoup d'autres pourraient venir étayer, révèle selon moi une tendance de fond. Les magistrats veulent sévir contre les individus qui abusent de la crédulité des enfants et souillent "l'innocence ». ${ }^{(25)}$

\section{* Le regard des médecins}

La décision de faire pratiquer une expertise médico-légale et éventuellement une ou plusieurs contre-expertises est laissée à la discrétion du seul juge d'instruction. Il n'est pas tenu légalement d'y recourir. ${ }^{(26)}$ Il ressort des affaires étudiées que l'expertise médicale n’est en général 
requise par le juge d'instruction qu'à partir du moment où il présume qu'il y a pu y avoir viol. Tout le travail des médecins consiste dès lors à établir si une présomption s'avère fondée. Leurs conclusions sont consignées dans des rapports d'expertise, conservés dans les dossiers criminels. Ces expertises sont pratiquées dans 13 affaires sur un total de 30, mais, dans certains dossiers "délicats", plusieurs praticiens sont appelés à confronter leurs vues. À une exception près, on peut dire que la psychologie de la victime, les troubles psychiques ressentis par elle après l'agression en particulier, ne sont jamais pris en compte. Les observations des médecins se limitent aux seules lésions corporelles. À la lecture des expertises médicales, on est frappé par la circonspection des médecins et plus encore par la teneur des conclusions de certaines, clairement "orientées" par des préjugés relatifs à l'enfance ou à la femme, a priori suspectes, pour ne pas dire coupables. Si bien que, comme le souligne J.-C. Martin, certaines de ces expertises deviennent parfois " de facto, selon le jugement, voire de par les insinuations des praticiens, de véritables actes d'accusation envers une victime ". ${ }^{(27)}$ Cependant les prudentes expertises ne constituent pas en soi des preuves légales et il n'est pas rare que les procureurs s'écartent dans leur qualification des faits des présomptions des praticiens.

Le 19 juin 1861, Joséphine Albert (12 ans) se présente chez le médecin du village, accompagnée de sa marraine. L'enfant raconte que, depuis le début du mois d'avril, son père commet régulièrement sur elle des abus sexuels. De son examen, Louis Sadonneux conclut que les parties génitales de l'enfant ont été "soumises à des violences ou à des manouvres à une époque rapprochée qui ont amené les désordres qui constituent la défloration ". Le médecin a en effet constaté que la vulve de Joséphine était de couleur brunâtre et échymosée, que les grandes lèvres étaient très écartées et tuméfiées, que l'hymen de l'enfant avait disparu. Le docteur termine en notant qu'il ne peut "toutefois affirmer que ces désordres ont été produits uniquement par des tentatives de viol ". ${ }^{(28)}$ La lecture d'un second rapport d'expertise (réalisé à la demande du juge d'instruction cette fois ${ }^{(29)}$ ) nous permet de comprendre à quoi le médecin fait allusion dans cette dernière phrase. Quinze jours après sa première visite, l'enfant se porte mieux. Les grandes lèvres ne sont plus tuméfiées et l'orifice du
Bissot, le 6 août 1849 .

(26) C'est aux

articles 43 et 44 du code

d'instruction criminelle qu'il est fait mention du recours éventuel aux experts pendant l'instruction.

(27) Jean-Clément

MARTIN, op. cit., p. 646.

(28) PJN, CAN, affaire Albert, le 19 août 1861; rapport médical de Louis Sadoneux, médecin à Spy, en date du 19 juin 1861.

(29) Entre-temps, la justice a été alertée. 
(30) Rapport médical du médecin Sadoneux du 5 juillet 1861 . Vu que le père de l'enfant est en détention provisoire et que l'état de l'enfant s'améliore, le médecin identifie donc bien les violences du père comme étant seules à l'origine des " désordres » qu'il a observé lors de sa précédente visite. La cause est « neutralisée ", l'effet tend à disparaître.

(30) Il publie à

Lausanne, en 1760, son ouvrage, De l'onanisme, version française largement augmentée d'un opuscule publié en latin en 1758 .

(31) Voir notamment : Elisabeth CLAVERIE,

«De la difficulté de faire un citoyen : les " acquittements scandaleux » du jury dans la France provinciale du XIXe siècle", Etudes rurales, $\mathrm{n}^{\circ}$ 95-96, Paris, juill.-déc. 1984, pp.143-165. Yves POURCHER, «Des vagin s'est rétréci. Tout ceci amène l'homme de l'art à conclure que "les désordres tant généraux que locaux observés le 19 juin ne sont nullement l'effet d'habitudes solitaires mais sont le résultat de violences exercées sur la personne de cette fille ". ${ }^{(30)}$ Le médecin soupçonnait donc que les graves lésions qu'il avait observées chez la fillette aient pu provenir d'habitudes de masturbation. Par delà le fait que cela apparaît comme une aberration en terme de diagnostic médical, il faut relever qu'il y a, à l'origine de cette présomption, le préjugé fortement ancré au XIXe siècle de "l'enfant pervers", s'adonnant aux "pratiques solitaires". Il n'est pas tellement étonnant de retrouver cette obsession de la masturbation enfantine dans les conclusions de l'expertise médicale du docteur. En effet, l'" onanisme comme maladie grave menant inexorablement à la folie ou à la mort » est une invention médicale du XVIIIe siècle, due entre autre à un médecin suisse, le docteur Tissot. ${ }^{(31)}$ Ses idées se seraient rapidement répandues dans la seconde moitié du XVIIIe siècle et la première du XIXe siècle, par le biais d'un nombre impressionnant de traités alors édités en France et en Belgique sur le sujet. J'ajoute que, malgré un rapport d'expertise finalement accablant pour le prévenu et portant à penser que le viol a été consommé (l'enfant a en effet été "déflorée"), Albert n'est finalement déclaré coupable "que" d'" attentat à la pudeur consommé avec violence sur sa fille légitime "; l'accusé est disculpé du chef de viol par les jurés. Le fait qu'il s'agisse d'un crime d'inceste n'y est sans doute pas étranger, mais j'y reviendrai ultérieurement.

\section{* Magistrats et jurés : des sensibilités différentes}

La coexistence au sein d'une même juridiction (la cour d'assises) de juges professionnels et de juges d'une session (les jurés) ne va sans créer certaines tensions, problème à vrai dire toujours d'actualité. Pour le XIXe siècle, plusieurs historiens se sont déjà livrés à une étude de ces différences de sensibilités qui opposent jurés et magistrats dans la perception des faits qu'ils ont à juger. ${ }^{(32)}$ Deux législations ont été mises en vigueur en Belgique entre 1830 et 1867 ayant comme but de permettre une "réappropriation" de l'exercice de la justice par les magistrats. Il s'agit des dispositions légales de 1838 et de 1849 dont l'objectif est principalement de lutter contre ce que les magistrats dénonçaient comme 
les « acquittements scandaleux " du jury. ${ }^{(33)}$ L'arrêté du 15 mai 1838 et la loi du 15 mai $1849^{(34)}$ contiennent des dispositions qui permettent la correctionnalisation des crimes avec, de l'une à l'autre, une latitude de plus en plus en grande accordée aux magistrats. ${ }^{(35)}$ Traduits en correctionnelle en vertu de circonstances atténuantes, les prévenus ne peuvent plus bénéficier de l'éventuelle clémence d'un jury. ${ }^{\left({ }^{36}\right)}$ Il s'agit bien là d'un "mécanisme de renforcement de la répression ". ${ }^{(37)}$ L'arrêté du 15 mai 1838 présente un second volet, lui aussi destiné à limiter les divergences de points de vue entre magistrats et jurés. Y sont prévues des mesures qui durcissent les critères d'accès à la fonction de juré, afin - en principe de ne conserver sur le banc du jury que des "élites" partageant une sensibilité commune avec les magistrats. Si ces nouvelles législations portent dans une certaine mesure leurs fruits, ${ }^{(38)}$ la "représentation consensuelle de l'équité " ${ }^{(39)}$ recherchée n'en est pas pour autant atteinte.

Avant d'aborder l'étude d'une affaire d'inceste à ce propos particulièrement édifiante, je livrerai quelques observations générales. Je dirais que les jurés semblent plus enclins que les magistrats à mettre entre parenthèses l'acte de violence sexuel pour se focaliser beaucoup plus sur un jugement de son auteur. Sans doute peut-on dire que les jurés font prévaloir, à l'inverse des magistrats, un ordre "plus masculin que moral ". ${ }^{(40)}$ L'analyse des cas d'acquittement montre à quel point le profil de l'accusé peut avoir une incidence sur le verdict que rendent les jurés. Les six prévenus déclarés innocents par le jury ont un casier judiciaire vierge. Il bénéficient en plus d'une très bonne réputation, et, pour certains, de solides appuis. Aussi, sans vouloir généraliser, lorsque les jurés doivent rendre leur verdict dans des questions de violence sexuelle, on peut avancer que c'est avant tout un individu, l'accusé, qui est jugé. Pour peu que l'accusé bénéficie d'un profil favorable, les jurés sont beaucoup plus réticents à le condamner, c'est-à-dire, en somme, à le bannir socialement. S’il est faux de dire que les magistrats parviennent à déconnecter dans le jeu des débats l'acte délictueux d'avec son auteur, ils y parviennent cependant beaucoup mieux que les jurés. À la différence de ces derniers, les magistrats sont moins prioritairement sensibles au profil des accusés. Ils sont d'abord attentifs à la nécessité de réprimer l'auteur d'un acte assises de grâce "? Le jury de la cour d'assises de la Lozère au XIXe siècle ", Etudes rurales, $\mathrm{n}^{\circ}$ 95-96, Paris, juill.-déc. 1984, pp. 167180.

(32) Il était courant que les jurés déclarent innocents des prévenus pourtant manifestement coupables, parce qu'ils encouraient une peine que le code pénal de 1810 rendait disproportionnée par rapport à la gravité de leurs actes.

Voir, à ce propos, Elisabeth CLAVERIE, op. cit., p. 143.

(33) Arrêté du 15 mai 1838, Pasinomie, 3ème série, t. VIII, 1838, pp. 63-74; loi du 15 mai 1849, Pasinomie, 3ème série, t. XIX, 1849, pp. 210-211.

(34) La loi du 15 mai 1849 permet la correctionnalisation des crimes " dans tous les cas où ily a lieu de le faire ", tandis que celle du 15 mai 1838 ne l'autorise que 
pour les crimes frappés de la seule réclusion (viols ou attentats à la pudeur commis avec violence sur des personnes de plus de 15 ans). Loi du 15 mai 1849, Pasinomie, 3ème série, t. XIX, 1849, pp. 209-214.

(35) En correctionnelle, le jugement est rendu uniquement par des magistrats professionnels.

(36) M. Van de KERCHOVE, Le Droit sans peines. Aspects de la dépénalisation en Belgique et aux Etats-Unis,

Publications des facultés universitaires SaintLouis, Bruxelles, 1987, p. 29.

(37) La tendance à la correctionnalisation des crimes de violences sexuelles se généralise, surtout après 1849 . Il est manifeste que la correctionnalisation des crimes "porte ses fruits", puisque, avant 1849 , une affaire sur trois se immoral, selon une répression (une peine) qui sera d'autant plus importante que le crime est perçu comme témoignant d'une grande immoralité. Les magistrats semblent moins sensibles au profil de l'accusé, à sa réputation qu'au degré d'immoralité manifesté à travers son crime.

La réforme législative durcissant les critères d'accession à la fonction de juré ne semble pas avoir apporté les résultats escomptés. Celle-ci était censée placer désormais aux côtés des magistrats des hommes de leur niveau d'instruction, "des élites aux convictions unanimes marquées du sceau d'un intérêt général, unifié, homogène, détaché des investissements du particulier » et des valeurs locales. ${ }^{(4)}$ Mais les "parités idéologiques" ne semblent pas encore atteintes. Les différences de sensibilité demeurent.

Deux chefs d'accusation sont retenus à charge de Théodore Lambert, ${ }^{(2)}$ un ouvrier terrassier de 36 ans n'ayant pas d'antécédents judiciaires : il est d'une part accusé d'avoir " commis pendant le mois d'avril 1862 à diverses reprises des attentats à la pudeur consommés ou tentés avec violence » sur sa fille légitime âgée de 13 ans, Adèle. Il est également accusé d'avoir "volontairement porté des coups et fait des blessures à sa dite fille ", le 28 avril 1862. Le 18 août 1862, le jury namurois déclare Lambert coupable du second chef d'inculpation mais le disculpe du premier, lui faisant éviter de ce fait une peine forcément sévère ${ }^{(4)}$ : sur la base de ce verdict, les magistrats de la cour d'assises condamnent Lambert à un an de prison (pour son inculpation pour coups et blessures). Particularité de l'affaire, on trouve dans le dossier une note manuscrite, probablement rédigée par le président de la cour. Il s'y exprime sur le verdict rendu par le jury et justifie la durée de la peine dont est frappé Lambert : «cette peine se ressent, on ne peut se le dissimuler, de la conviction qui animait la Cour de la culpabilité de l'accusé sur le premier chef d'accusation. L'erreur du jury est manifeste à mon sens et je l'ai déjà signalé dans le compte rendu des assises de cette session. ${ }^{(4)}$ Aussi, j'entends que la peine d'un an d'emprisonnement du chef de coups doit être maintenue ". Apparemment la cour considérait que, compte tenu des éléments réunis par l'instruction, le doute n'était pas permis quant à la culpabilité de Lambert sur le chef des violences sexuelles. Pour compenser dans une certaine mesure l'injustice du verdict rendu, les magistrats condamnent Lambert à un an de pri- 
son. Cette inhabituelle sévérité pour ce genre de faits suscite apparemment des protestations, très compréhensibles dans un contexte juridique où la correction paternelle est un droit légitime.

De même, le verdict rendu par le jury - verdict "scandaleux", selon le juge - doit-il être compris dans un contexte particulier. Outre le fait que le jury cherche peut-être à soustraire Théodore Lambert à une peine forcément très lourde, il est probable que les affaires d'inceste suscitaient chez les jurés, à cette époque, un réel malaise, en ce sens qu'elles les confrontaient à des sentiments mélangés et difficilement conciliables : d'une part, chez certains tout au moins, la révolte face à des actes ignobles; d'autre part, peut-être aussi une certaine "compassion" envers les accusés, forgée dans une secrète identification au père de famille qu'ils étaient pour la plupart et contre lequel se retournaient l'épouse et la fille, ce qui allait à contresens de la conception du modèle familial d'alors. Enfin, dans le contexte d'une société très patriarcale, où la figure du pater familias est tout à fait dominante au sein du modèle familial, il va sans dire que le poids de la parole d'une fillette contre celle de son père est plus que relatif.

\section{3. - LE REGARD DE LA SOCIÉTÉ. PERCEPTIONS DES VIOLENCES SEXUELLES COMMISES SUR ENFANTS DANS LE MONDE RURAL NAMUROIS : LES LIMITES DE LA TOLÉRANCE}

Après avoir examiné la manière dont le législateur, les magistrats et les jurés percevaient les actes de violence sexuelle commis sur enfants, arrêtons-nous maintenant au regard porté sur ceux-ci par les acteurs du monde rural. Les réactions villageoises face à la violence sexuelle sont fort diverses. Leur variété n'empêche cependant pas une certaine catégorisation. Dans le monde rural namurois, les réactions sociales face à l'acte de violence sexuelle vont d'une relative tolérance à un rejet plus ou moins marqué. Ces réactions sont bien sûr tributaires d'un ensemble de facteurs : elles varient fortement - entre autres - en fonction du profil (âge, réputation, position dans l'échelle sociale) aussi bien de l'agresseur que de la victime et en fonc- solde par un acquittement, contre une sur six après 1849 .

(38) Elisabeth CLAVERIE, op. cit., p. 146.

(39) Jean-Clément MARTIN, op. cit., p. 647.

(40) Elisabeth CLA-

VERIE, op. cit., p. 148.

(41) PJN, CAN, affaire Lambert, le 18 août 1862.

(42) Un an plus tôt en effet, Albert est condamné par les magistrats namurois à une peine exemplaire pour des faits similaires, celle des travaux forcés à perpétuité, bien que le verdict rendu par les jurés soit très « prudent ".

(43) PJN, CAN affaire Albert, le 19 août 1861.

(44) Ce document, qui était transmis au ministre de la justice, permettait aux présidents des tribunaux de signaler les éventuels dysfonctionnements de l'appareil judiciaire.

(45) PJN, CAN, affaire Daiche, le 6 février 
1834. Pierre Daiche a été condamné, mais par contumace, c'est-à-dire que le verdict a été rendu en son absence (il est alors fugitif) par des magistrats. Il eût fort probablement été acquitté par un jury.

(46) En tant que notabilités locales, les bourgmestres participent bien souvent au processus d'arrangement, ou du moins le tolèrent, ne s'acquittant qu'avec difficulté de leurs obligations envers l'appareil judiciaire. Cette attitude des bourgmestres prend sa source dans une volonté de préserver la « concorde sociale "; de plus, en agissant de la sorte, ils évitent d'éventuelles rancunes à leur égard (qui pourraient émaner de la famille de l'accusé et même de la communauté rurale, si l'accusé est bien en vue); il s'agit encore également pour eux de conserver une " certaine tion de la nature de l'abus sexuel commis. Le profil de l'auteur de l'abus sexuel est malgré tout le facteur sans doute le plus déterminant.

Lorsque l'accusé est nanti d'un profil avantageux, on peut observer à son égard, au sein de la communauté villageoise, un certain nombre d'attitudes que l'on pourrait définir comme des "protections", - pour reprendre un vocable très en vogue aujourd'hui en Belgique. Ces réactions revêtent cependant des formes fort différentes selon que la victime est une jeune femme ou une enfant.

Le 23 juin 1833, Pierre Daiche, ${ }^{(4)}$ un domestique de 25 ans, tente de violer Victoire Paquet, une servante de 21 ans. Un domestique travaillant avec la victime témoigne avoir vu l'agresseur quelques minutes après le retour de Victoire (tout essoufflée et dont les effets sont dérangés), le visage égratigné, mais poussant la chansonnette, considérant apparemment son forfait comme une plaisanterie. La victime ne porte pas plainte d'elle-même (ce qui, dans le monde rural, est de toute façon rarissime), mais - peut-être s'agit-il d'une vengeance - quelqu'un se décide à avertir la justice. Deux gendarmes en tournée certifient dans un procès-verbal daté du 28 juin avoir été informé "par la rumeur publique ", lors de leur tournée à Corroy-le-Château, d'une tentative de viol commise par un certain Pierre Daiche. Le dénonciateur choisit donc de s'adresser aux gendarmes comme relais à la justice, plutôt qu'au bourgmestre, trop impliqué dans la vie villageoise et qui n'aurait sans doute pas ménagé ses efforts pour étouffer l'affaire d'une manière ou d'une autre. ${ }^{(4)}$ On retrouve en effet dans le dossier de l'affaire trois lettres envoyées par des "personnalités" au président de la cour d'assises de Namur. Ces documents constituent des sources fort précieuses en ce sens qu'elles nous mettent en prise directe avec le discours des notabilités rurales sur les rapports hommes-femmes et sur la sexualité en général. Le bourgmestre de Cortil-Noirmont déclare dans sa lettre au président de la cour d'assises, sur un ton où se mêlent condescendance et paternalisme : "en considérant la classe de la société à laquelle appartiennent ce garçon et cette fille, ily a lieu à examiner si ce que l'on dit s'être passé entre eux peut vraiment être considéré comme un attentat à la pudeur ou plutôt être envisagé seulement comme une de ces licences si ordinaires dans les cabarets de cam- 
pagne ». Un gros cultivateur du village, également conseiller communal, explique, quant à lui, que Daiche était quelque peu ivre lorsqu'il rencontra la plaignante et qu'il "ne doit s'être permis que ce que font tous les paysans et les paysannes si souvent sans que celles-ci s'en fâchent". Un autre fermier important déclare enfin que, si Daiche "s'est permis une petite licen$c e$ " envers Victoire, cela ne peut être attribué " qu'aux anciennes liaisons qu'il doit avoir eues dans le temps avec elle " et à son ébriété. Il ajoute que, dès lors, "tout autre motif de persister dans cette plainte si injuste ne pourrait être considéré que comme guidé par des sujets de jalousie ou de vengeance ». Les appuis dont bénéficie Daiche ne se limitent pas aux quelques lettres envoyées par des notabilités locales : plusieurs procès-verbaux de gendarmes témoignent d'importantes difficultés pour récolter des renseignements relatifs à la fuite du prévenu. La population du village s'enferme dans le silence, cherchant à protéger Daiche. Certains villageois disent aux gendarmes que la victime ne peut être "qu'une femme de mauvaise vie " pour avoir fait inculper le "brave garçon".

Dans cette affaire, ${ }^{(47)}$ le prévenu est célibataire, encore jeune et issu certes d'un milieu social modeste, mais disposant de nombreuses relations, d'une bonne réputation (jusque-là) et d'un solide "capital-sympathie". La victime est aussi une jeune fille célibataire, néanmoins en âge de se marier, appartenant également à un milieu modeste. L'agression sexuelle a été violente, mais le viol n'a apparemment pu être consommé. Dans de telles affaires, l'agresseur bénéficie d'une certaine bienveillance de la communauté, masculine du moins, ce qui le conforte souvent dans une attitude d'apparente insouciance par rapport à l'acte perpétré. Sans doute parce que les actes de violence sexuelle de ce genre sont perçus comme une expérimentation relativement tolérée, dans la mesure où son auteur n'est pas promis à une proche union vu son état d'indigence (il dispose de ce fait, "en compensation ", d'une plus grande liberté) et qu'il ne s'est pas immiscé, par ses "jeux", dans "les rivalités matrimoniales en cours ". ${ }^{(48)}$ Aussi, pour peu que la justice mette en examen le "séducteur" violent, les langues se délient et les solidarités masculines se manifestent avec véhémence, mues par l'indignation. La victime est perçue comme une femme scandaleuse qui cherche à nuire mainmise sur les litiges

locaux ». Pierre-Yves

GUILARD, « Les

Pratiques infrajudiciaires des communautés villageoises Côte-d'Oriennes du XIXe siècle ", in Benoit GARNOT (Dir.), L'Infrajudiciaire du Moyen Age à l'époque contemporaine. Actes du colloque de Dijon, 5-6 octobre 1995, Publications de l'université de Bourgogne, Dijon, 1996, t. LXXXI, p. 434.

(47) L'affaire

Douxchamps (PJN,

CAN, affaire

Douxchamps, le 26 août 1856) est une autre manifestation éclatante de ces «solidarités viriles». Douxchamps (32 ans), accusé d'avoir tenté de violer une jeune fille de 17 ans, semble être soutenu par pratiquement tous les hommes de sa commune. Une lettre visant à le disculper et envoyée au procureur de Roi prend quasiment des allures de 
pétition, dès lors qu'y sont apposées plus de 110 signatures.

(48) Pierre LAMAISON, Elisabeth CLAVERIE, L'impossible mariage : violence et parenté en Guévaudan aux 17ème, 18ème et 19ème siècles, Paris, Hachette, 1982, p. 208. "sans raison", si ce n'est sans doute par strict désir de vengeance. Il est intéressant de remarquer que certaines des lettres de notables analysées reconnaissent presque implicitement l'agression sexuelle commise. Seulement, précisément, pour ces hommes qui se mobilisent, l'acte en lui-même n'est pas grave. Il ne s'agit que d'une "petite licence ".

On peut se demander si les violences sexuelles commises sur enfants suscitent en général plus d'indignation (et sont donc moins "tolérées") dans la communauté rurale que si elles sont commises sur des jeunes femmes ou des femmes adultes. Pour apporter réponse à pareille question, un élément semble assez significatif : quand l'agression sexuelle est commise sur enfant, jamais les véritables démonstrations de solidarités masculines rencontrées dans le cas précédemment évoqué n'apparaissent. Les agressions sexuelles commises sur enfants ne sont sans doute pas perçues comme peuvent l'être celles qui sont exercées sur des jeunes femmes célibataires, à savoir, comme de "vilains jeux sans réelle gravité". Cependant le statut du "pédophile" peut générer des réactions très ambiguës, tant au sein des familles des victimes que parmi la communauté rurale. Examinons l'affaire Gaspart dans laquelle le pédophile est aussi l'instituteur du village, fonction qui lui confere, dans une petite commune rurale du moins, un incontestable prestige social.

Thérèse Mouchette (12 ans) est régulièrement victime d'abus sexuels commis par Gaspart, après la classe, durant l'année 1856. Lorsque sa famille l'interroge sur ses retards, Thérèse répond que l'instituteur la garde après la classe, pour préparer sa première communion. Sa grandmère à qui elle se confie est sous le choc. Quand l'oncle et la tante de la victime apprennent que l'instituteur " a pris des libertés " sur leur filleule, ils sont atterrés et évitent désormais d'aborder le sujet. Cependant, la tante de la victime révèle quelques jours plus tard toute l'affaire aux Delvosal, fermiers semble-t-il assez aisés chez lesquels elle travaille. La nouvelle se répand rapidement à travers le village. Par quelle filière, il est comme toujours impossible de le dire; mais bientôt le curé de la paroisse désire voir l'enfant. L'épouse Delvosal qui prend pratiquement l'affaire en main dès ce moment accompagne Thérèse chez lui. Le religieux s'adresse à la victime en ces termes : "étiez-vous contente de vous lais- 
ser faire de vilains jeux comme ça?». En filigrane de cette interrogation, ressort le thème de l'enfant suspecte. Le curé semble presque accuser l'enfant d'avoir pris goût à ces "vilains jeux". Dans l'esprit de cet homme, l'enfant est sans doute déjà en partie coupable, par le seul fait d'être entrée en contact avec une "sexualité sacrilège". Lorsqu'ils l'interrogent, le curé et la fermière ne poussent pas trop loin les questions, parce que (selon la fermière) le curé et elle comprennent "très bien de quoi il s'agit». Le sujet crucial, le sexe "infamant", est toujours pudiquement évité.

L'instituteur Gaspart se rend chez les tuteurs de Thérèse en leur promettant une récompense, s'ils jurent de "se taire ». Devant leur réaction face aux révélations de l'enfant et malgré la démarche de la tante de Thérèse, il n'est pas difficile de comprendre à quel point la famille de la petite fille répugnerait bien certainement à entamer des poursuites contre l'instituteur. D'une part, en raison de leur crainte d'être comme " contaminés par l'infamie " rendue publique; d'autre part, étant donné l'excellente réputation de Gaspart, dont la respectabilité impressionne probablement la modeste famille. Aussi la justice va-t-elle se saisir de l'affaire sans doute bien malgré eux. En témoigne la réaction de la grand-mère de la victime qui, interrogée dans le cadre de l'instruction de l'affaire, ira jusqu'à tout nier. La responsabilité de la poursuite incombe en fait aux Delvosal. Sans eux, il est fort probable que Gaspart serait parvenu à ses fins et que l'instituteur n'aurait pas été confronté à la justice. Certes son crime ne serait pas demeuré inconnu, mais il serait sans doute resté secret, dans la mesure où une pression sociale ou « un accord tacite aurait imposéle silence au village $"{ }^{(4)}$ Seulement, Xavier Delvosal veut que Gaspart soit jugé. Il met un négociant de Ciney au courant des faits et lui demande d'en informer la justice. Si le fermier ne le fait pas lui-même, c'est, explique-t-il, parce qu'il "craint les rancunes et le feu». Cette phrase lourde de sens témoigne bien de ce qu'une partie au moins de la communauté rurale reste attachée à la cause de l'instituteur, même si aucun autre document du dossier n'en dit davantage sur ce point. La peur d'une vengeance privée est en tout cas présente chez le cultivateur.

Si l'indignation que suscite la violence sexuelle commise sur enfant est bien réelle, elle s'accompagne bien souvent, lorsqu'elle est révélée,

(49) Pierre-Yves GUILARD, op. cit., p. 431. 
(50) PJN, CAN, affaire Bissot, le 6 août 1849 .

(51) Anne-Marie

SOHN, « Les Attentats

à la pudeur sur les

fillettes en France (1870-

1939) et la sexualité quo-

tidienne ", in Alain

CORBIN (Prés.),

Mentalités. Histoire des

cultures et des sociétés,

Violences sexuelles, Paris, 1989, p. 98.

(52) Ibid., affaire

Gauthier, le 3 avril 1837.

(53) Georges VIGA-

RELLO, Histoire du viol, XVI-XXe siècles, L'univers historique, Paris 1998, p. 8.

(54) Ibid., affaire Cousin, le 20 août 1840. d'un violent rejet, parce qu'en tant que sexualité sacrilège elle est frappée d'un véritable tabou. La difficulté est grande pour de modestes familles de se retourner contre des pédophiles dont le statut social impressionne, et ce d'autant plus qu'elles sont d'origine modeste. La réaction de la grand-mère dans l'affaire Gaspart n'est pas un cas isolé. Dans l'affaire Bissot, ${ }^{\left({ }^{50}\right)}$ curé qui a commis des attouchements sur plusieurs petits garçons, lors de l'instruction, les mères des enfants tentent de les amener à nier la triste vérité, afin, avouera l'une d'elles plus tard au juge d'instruction, "de ne pas faire de peine au curé ». À nouveau, il y a une volonté d'occulter l'infamie, tout en cherchant également à protéger un personnage que l'on respecte et avec lequel se sont tissés des liens amicaux. Il y a chez ces mères comme une "révérence sociale " paralysante. Dans cette même affaire, les pères des garçons réagissent presque tous de la même manière : une fois informés, ils exigent que l'on n'en parle jamais plus en leur présence. Dans l'affaire Gauthier, ${ }^{(52)}$ jeune religieux qui commet divers attouchements sur une vingtaine de garçons de Beauraing, le cap de la sourde rancune est dépassé lorsque le bénédictin quitte la commune précipitamment, pressentant sans doute le danger. Le lendemain même de son départ, son ermitage est incendié. Seulement alors s'exprime la vindicte de celui ou ceux qui depuis longtemps peut-être savaient, sans pouvoir ou vouloir agir.

Ce qui étonne le plus à l'analyse de certains dossiers, c'est la suspicion qui s'abat sur des enfants victimes, enfants "enveloppés », eux et leur agresseur, dans une même indignité coupable. ${ }^{(53)}$ L'affaire Cousin ${ }^{(54)}$ est à ce propos édifiante. Le 15 août 1840, Jean-Baptiste Cousin, un ancien garde-champêtre de 66 ans, invite Marie Collot et Félicie Godet (6 et 7 ans) à "aller se promener", leur promettant des couques et des sous. Une fois à l'écart, dans un verger, derrière une haie, Cousin abuse sexuellement des deux enfants. Pendant ce temps, Marie-Jeanne Jaspart observe sans être vue. Cousin tente, semble-t-il, de violer successivement les deux enfants, leur causant de fortes douleurs. Ce n'est apparemment qu'au bout de longues minutes que Jaspart finit par intervenir. À son arrivée et après l'avoir traité de " cochon ", Cousin se relève et tous trois s'éloignent. Après son intervention, Jaspart rentre à la ferme pour racon- 
ter le "dégoûtant spectacle » auquel elle a assisté. Jean-Baptiste Dassy a également vu Cousin avec les deux enfants. Dans sa déposition, il indique que Cousin était debout et les fillettes couchées par terre. Il passe son chemin, sans s'arrêter. Dassy a pourtant bien compris de quoi il s'agissait, comme en témoigne cette surprenante réaction : rencontrant sur sa route le père de Félicie, Dassy lui dit d'aller rechercher sa fille près du verger et de lui donner "une bonne volée, qu'elle l'a bien méritée ». Sans demander plus d'explications, Xavier Godet court vers le lieu dont a parlé Dassy. En chemin, il croise Marie-Jeanne Jaspart qui lui dit aussi d'aller récupérer sa fille "arrière de Cousin, arrière de ce vieux cochonlà ", pour lui donner (à sa fille!) " une bonne correction ». Il continue sa route et rencontre enfin Félicie qui "vient tomber à moitié morte " à ses pieds en s'écriant, "Mon Dieu papa!». Xavier Godet poursuit sa déposition en ces termes : «Me doutant bien qu'il s'était passé quelque chose d'extraordinaire entre ma fille et Cousin que Jaspart avait désigné de vieux cochon, je fessai ma fille ". Au même instant, le père de l'enfant aperçoit Cousin. Il l'attrape et lui donne un coup de poing qui le fait tomber. Cousin se relève et s'éloigne, Godet l'invective. Le père donne la fessée à sa fille et il frappe Cousin. L'un et l'autre sont en quelque sorte mis sur le même pied. C'est un peu comme si le père considérait Cousin coupable d'avoir fait goûter trop tôt sa fille à un sexe interdit et coupable sa fille d'y avoir goûté. Prend-il conscience du fait qu'il y a eu abus de pouvoir? Peut-être ne s'en donne-t-il pas la peine. Jaspart et Dassy considèrent tous deux qu'elle a mérité une correction et le père la lui donne. Comme dans l'affaire Gaspart où le curé soupçonne l'enfant d'avoir participé de plein gré aux «vilains jeux ${ }^{(55)}$ que lui impose l'instituteur, ces gens semblent ne pas parvenir à concevoir que ces enfants ont été abusées par un adulte qui a profité de leur incapacité à poser un consentement ou un refus. La notion de discernement et son contraire leur est probablement étrangère. De plus, il semble que, pour ces gens, l'enfant soit a priori coupable.

Dans sa déposition, le père de Félicie ajoute qu'après l'agression de sa fille il n'a pas voulu lui demander d'explications, " car cela le répugnait ». Cette attitude du père de Félicie présente des similarités avec le com-

(55) Je reprends ses termes. 
(56) Dans 17 des 30 affaires analysées, l'action en justice est menée suite à une plainte de la famille de la victime, plainte la plupart du temps (10 cas sur 17) déposée chez le bourgmestre du village, " autorité » la plus proche et la plus accessible pour des familles qui ne maîtrisent pas l'écrit.

(57) PJN, CAN, affaire Servais, le 27 février 1844. portement de l'oncle de Thérèse dans l'affaire Gaspart, celui des pères des enfants dans l'affaire Bissot. À la tristesse et à la colère se superpose le violent rejet d'une réalité, le sexe, qui lui fait honte, "le répugne" même et qu'il faudrait définitivement enterrer, cacher aux yeux de tous, parce que révélée publiquement, elle jetterait son infâmie sur la famille entière. De façon générale, je dirais que les pères, davantage que les mères, semblent envisager la gravité de l'acte de violence sexuelle avant tout en terme d'atteinte causée à l'honneur familial.

Pour en terminer avec ce point, il me faut maintenant parler d'affaires où les faits de violence sexuelle commis suscitent des réactions - tant dans les familles des victimes que dans la communauté villageoise - très différentes de celles qui ont été étudiées. Il est, d'une part, difficile de ne pas voir dans certaines dépositions des pères ou des mères des victimes une apparente indifférence par rapport à l'acte commis, pour autant que ses conséquences soient - au moins superficiellement - anodines. Des parents avouent "ne s'être pas préoccupés autrement " des pleurs de leur enfant victime d'attouchements, parce que, une fois remis de son émotion, il semblait en bonne santé. Indifférence et/ou résignation peut-être par rapport à des pratiques jugées banales dans un quotidien fait de violence. D’autre part, les cas que j’ai évoqués pour leur spécificité ne doivent pas occulter les affaires où l'indignation des familles n'est pas suivie d'un "rejet", d'une volonté d'occulter les faits, mais au contraire d'une action en justice dont elles sont l'instigatrice. ${ }^{(56)}$ Dans l'affaire Servais, ${ }^{(57)}$ Nicolas Michaux, le père de la victime se rend chez le bourgmestre pour porter plainte, le jour même du viol commis sur sa fille de 10 ans. Le bourgmestre va voir la victime qui, comme il l'indique dans son procès-verbal, est "beaucoup détruite et ensanglantée ". La gravité des blessures occasionnées par le viol explique la promptitude de cette réaction, mais ce n'est pas tout. Servais n'est ni quelqu'un de spécialement bien en vue au village, ni quelqu'un d'influent. Son intégration dans la communauté est très relative. À nouveau, le profil de l'accusé est déterminant. La plupart du temps, les bourgmestres ne transmettent directement les informations dont ils disposent au procureur que si l'agresseur sexuel est de mauvaise réputation ou marginalisé par 
un comportement asocial. Sinon ils auront tendance à privilégier l'arrangement au sein de la communauté villageoise.

\section{CONCLUSIONS}

Face aux violences sexuelles, les réactions sociales dans le monde rural namurois sont très contrastées. Certaines de ses formes revêtent, pour les hommes du moins, le caractère de "petites licences" sans gravité : il s'agit probablement avant tout des agressions sexuelles (tentatives de viol) commises par des hommes jeunes et célibataires sur des victimes adultes (ou ayant en tout cas dépassé le cap de l'enfance), elles aussi célibataires et d'origine modeste. Lorsque les auteurs d'actes de ce type bénéficient d'un "capital-sympathie" et pour peu que la justice s'empare de leur cas, les solidarités masculines "se déchaînent", mues par un profond sentiment d'indignation.

Si les magistrats ne sont nullement influencés par les démarches menées par les populations rurales pour disculper certains agresseurs, même quand elles le sont par des notabilités locales, ce n'est pas le cas des jurés. Lorsque de telles solidarités se sont manifestées dans la communauté rurale, le prévenu est toujours acquitté par les jurés. Cette sensibilité partagée est d'autant plus compréhensible que, faut-il le rappeler, le jury est composé exclusivement d'hommes. D'autre part, jusqu'en 1838, les jurys sont encore majoritairement composé de notabilités issues du monde rural. La loi de 1838 qui durcit les conditions d'accès à la fonction de juré et les lois de 1838 et de 1849 relatives à la correctionnalisation des crimes vont permettre aux magistrats d'accomplir ce qu'on pourrait nommer une "réappropriation de l'exercice de la justice ". Mais si le nombre d'acquittements est sensiblement en baisse dans les affaires de violence sexuelle jugées devant les assises namuroise, après 1849, la "représentation consensuelle de l'équité " ${ }^{(58)}$ escomptée n'est pas atteinte, malgré les dispositions légales qui ont modifié la composition du jury : entre les magistrats et les jurés, des différences de sensibilité subsistent. Ce décalage se manifeste particulièrement dans une affaire

(58) Elisabeth CLAVERIE, op. cit., p. 146. 
(59) Anne-Marie SOHN, op. cit., p. 98. d'inceste. Dans ce genre d'affaires, la réticence des jurés à condamner les pères incestueux est sans doute d'autant plus grande que les repères communs, les valeurs établies y sont bouleversés. Des enfants révèlent les abus d'un père, des femmes accusent leur mari.

Dans le monde rural, la tolérance semble moins jouer en faveur des auteurs d'actes de pédophilie. En témoigne en tout cas le fait que, dans aucun dossier de ce genre, je n'ai observé les véritables élans de "solidarités viriles” témoignés à l'égard de certains prévenus ayant tenté de violer une femme adulte. Il est cependant difficile de généraliser : ce genre d'actes ne suscitent pas que de l'indignation. À l'analyse des réactions des familles des victimes, en effet, on remarque qu'il n'est pas rare que la révélation de la violence sexuelle ne suscite, apparemment, que de l'indifférence, surtout lorsqu'il n'y a pas eu de véritable recours à la violence. À l'opposé, les réactions les plus extrêmes, soit dans la crainte du scandale, soit dans la colère et l'humiliation qu'engendre la révélation de l'abus sexuel, se rencontrent toujours lorsque le pédophile est auréolé d'une sorte de prestige moral attaché à sa fonction sociale. Mais lorsqu'un pédophile est nanti d'un tel statut, la désapprobation ou l'indignation que suscitent ses actes dépasse difficilement le cap de la sourde rancune. Dans ce genre d'affaires, les familles des victimes répugnent, semble-t-il, à porter plainte contre ces individus. À l'origine de ces attitudes, il y a, surtout chez les pères de famille, un profond rejet de révélations qui font honte et que l'on ne veut dès lors pas rendre publiques à travers une action en justice; également une certaine "révérence sociale" ${ }^{(59)}$ envers un personnage que l'on respecte. Cette révérence sociale est d'autant plus marquée que les familles des victimes sont d'une origine modeste.

Du "discours" des accusés à celui des médecins, des propos des notabilités rurales à ceux de certains députés, on retrouve le thème d'une victime a priori suspecte, entachée du soupçon qui pèse sur elle d'avoir été, durant l'agression sexuelle, non pas victime, mais en réalité "secrètement consentante". Pour peu que j'aie pu en juger, les magistrats namurois ne sont apparemment que fort peu influencés par ce genre de "fantasmes", que ce soit durant l'instruction, dans l'exercice de la qualification des faits ou au moment de rendre la sentence. Ce qui, pour nos 
sensibilités contemporaines, apparaît comme le plus heurtant (parce que sans doute le plus inactuel), c'est la confusion, manifeste parfois, entre l'enfance victime et l'enfance coupable. Lors de leur examen des parties génitales d'enfants agressées sexuellement, plusieurs médecins attribuent l'origine des rougeurs, voire des blessures observées à l'onanisme. Parmi les réactions villageoises, plusieurs témoins ont semblé suspecter des enfants d'avoir participé de plein gré à des actes dont ils étaient seulement victimes, même s'ils n'y ont pas forcément opposé une résistance physique. À l'origine de ces réactions, il semble y avoir une incapacité à concevoir le non discernement de l'enfant et/ou son incapacité à poser un libre consentement. De plus, en ayant " participé " à une sexualité sacrilège (la conscience du "péché de la chair" est apparemment très forte chez ces gens), c'est un peu comme si ces enfants avaient été "enveloppés", eux et leur agresseur, dans une même indignité coupable. ${ }^{(60)}$

(60) Voir à ce propos Georges VIGARELLO, op. cit., p. 8. 